\title{
A FUNCTIONAL EQUATION RELATED TO THE PRODUCT IN A QUADRATIC NUMBER FIELD
}

\author{
LUCIO R. BERRONE AND LUIS DIEULEFAIT
}

\begin{abstract}
The functional equation
$f\left(x_{1}, y_{1}\right) f\left(x_{2}, y_{2}\right)=f\left(x_{1} x_{2}+\alpha y_{1} y_{2}, x_{1} y_{2}+x_{2} y_{1}\right), \quad\left(x_{1}, y_{1}\right),\left(x_{2}, y_{2}\right) \in \mathbb{R}^{2}$ arises from the formula for the product of two numbers in the quadratic field $\mathbb{Q}(\sqrt{\alpha})$. The general solution $f: \mathbb{R} \rightarrow \mathbb{R}$ to this equation is determined. Moreover, it is shown that no more general equations arise from a change of basis in the field.
\end{abstract}

\section{INTRODUCTION}

The functional equation in several variables

$$
f\left(x_{1}, y_{1}\right) f\left(x_{2}, y_{2}\right)=f\left(x_{1} x_{2}+y_{1} y_{2}, x_{1} y_{2}-x_{2} y_{1}\right),\left(x_{1}, y_{1}\right),\left(x_{2}, y_{2}\right) \in \mathbb{R}^{2}
$$

has deserved recently some attention in connection with the identity

$$
\left(x_{1}^{2}+y_{1}^{2}\right)\left(x_{2}^{2}+y_{2}^{2}\right)=\left(x_{1} x_{2}+y_{1} y_{2}\right)^{2}+\left(x_{1} y_{2}-x_{2} y_{1}\right)^{2} .
$$

The pair of algebraic identities given by (2) and by

$$
\left(x_{1}^{2}+y_{1}^{2}\right)\left(x_{2}^{2}+y_{2}^{2}\right)=\left(x_{1} x_{2}-y_{1} y_{2}\right)^{2}+\left(x_{1} y_{2}+x_{2} y_{1}\right)^{2}
$$

are known from ancient times. In the mathematical literature they were diversely named after mathematicians like Diophantus of Alexandria, Brahmagupta and Leonardo of Pisa who used them in their works (see, for instance, Proposition V at pg. 18 of [2]). When restricted to integer numbers both identities express the fact that the product of two sums of two squares is itself a sum of two squares. After a slight change in notation, (2) corresponds to the case $n=2$ in Lagrange's identity

$$
\left(\sum_{k=1}^{n} x_{k} y_{k}\right)^{2}=\left(\sum_{k=1}^{n} x_{k}^{2}\right)^{2}\left(\sum_{k=1}^{n} y_{k}^{2}\right)^{2}-\sum_{1 \leq k<j \leq n}\left(x_{k} y_{j}-x_{j} y_{k}\right)^{2},
$$

and can be also considered as a particular case of more general identities involving sums of squares such as the Euler's four squares identity. Furthermore, denoting by $i$ the imaginary unit, setting $z_{k}=x_{k}+i y_{k}, k=1,2$, and $\bar{z}=x-i y$ the

Key words and phrases. functional equation, additive and multiplicative function, quadratic field. 
conjugate of $z=x+i y$, identities (2) and (3) can be respectively written as particular instances of the functional relationships

$$
f\left(z_{1}\right) f\left(z_{2}\right)=f\left(\bar{z}_{1} z_{2}\right), z_{1}, z_{2} \in \mathbb{C}
$$

and

$$
f\left(z_{1}\right) f\left(z_{2}\right)=f\left(z_{1} z_{2}\right), z_{1}, z_{2} \in \mathbb{C} .
$$

Note that multiplicativity of the norm $f(z)=f(x+i y)=|z|=\sqrt{x^{2}+y^{2}}$ is expressed by (6), meanwhile invariance under conjugation is also involved in (5). Indeed, the solutions to the functional equation (6) are the morphisms of the multiplicative group $\langle\mathbb{C}, \cdot\rangle$ in the multiplicative group $\langle\mathbb{R}, \cdot\rangle$.

Now, for a square free integer $\alpha \neq 1$, consider the quadratic field $\mathbb{Q}(\sqrt{\alpha})$. In the basis $(1, \sqrt{\alpha})$, a generic member $\xi \in \mathbb{Q}(\sqrt{\alpha})$ is represented by $\xi=x+\sqrt{\alpha} y$, $x, y \in \mathbb{Q}$, and the product of two numbers $\xi_{i}=x_{i}+\sqrt{\alpha} y_{i}, i=1,2$ is given by

$$
\left(x_{1}+\sqrt{\alpha} y_{1}\right)\left(x_{2}+\sqrt{\alpha} y_{2}\right)=\left(x_{1} x_{2}+\alpha y_{1} y_{2}\right)+\sqrt{\alpha}\left(x_{1} y_{2}+x_{2} y_{1}\right) ;
$$

thus the functional equation

$$
f\left(x_{1}, y_{1}\right) f\left(x_{2}, y_{2}\right)=f\left(x_{1} x_{2}+\alpha y_{1} y_{2}, x_{1} y_{2}+x_{2} y_{1}\right),\left(x_{1}, y_{1}\right),\left(x_{2}, y_{2}\right) \in \mathbb{R}^{2}
$$

where $\alpha$ is a given real parameter naturally arises.

In Theorem 1 of [3], the general solution $f: \mathbb{R}^{2} \rightarrow \mathbb{R}$ of the equation (1) (or, equivalently, (5)) is shown to be of the form

$$
f(x, y)=\sigma(x, y) M\left(\sqrt{x^{2}+y^{2}}\right),
$$

where $M: \mathbb{R} \rightarrow \mathbb{R}$ is a multiplicative function and $\sigma: \mathbb{R}^{2} \rightarrow\{ \pm 1\}$ is a signum function. Recall that a function $M$ is said to be multiplicative when solves the multiplicative Cauchy equation

$$
M(x y)=M(x) M(y) .
$$

Similarly, every solution $A$ of the additive Cauchy equation

$$
A(x+y)=A(x)+A(y)
$$

is said to be an additive function. In the present paper, the following general result on equation (8) is established:

Theorem 1. The general solution $f$ of equation (8) depends on the signum of the parameter $\alpha$ and it is given by

$$
f(x, y)=\left\{\begin{array}{ll}
M_{1}(x+\sqrt{\alpha} y) M_{2}(x-\sqrt{\alpha} y) & \text { if } \alpha>0 \\
f=0 \text { or } f=1 & \text { if } \alpha=0 \\
M\left(\sqrt{x^{2}-\alpha y^{2}}\right) \exp (A(\operatorname{atan}(\sqrt{|\alpha|} y / x)) & \text { if } \alpha<0 .
\end{array}, \quad x, y \in \mathbb{R} .\right.
$$

In these expressions, $M, M_{1}$ and $M_{2}$ are arbitrary multiplicative functions and $A$ is an arbitrary additive function from $\mathbb{R}$ to $\mathbb{R}$. 
Among other results related to equation (8), a proof of Theorem 1 is developed along the following section. In the final section 3, several observations and remarks concerning equation (8) are made; particularly, a brief discussion is presented of the functional equations derived from the product formula in a quadratic field when the numbers in the field are expressed in other basis.

\section{Analysis of Equation (8)}

In solving equation (8), three essentially different cases arise depending on the signum of the parameter $\alpha$. In fact, for $\alpha=0$,equation (8) becomes

$$
f\left(x_{1}, y_{1}\right) f\left(x_{2}, y_{2}\right)=f\left(x_{1} x_{2}, x_{1} y_{2}+x_{2} y_{1}\right), \quad\left(x_{1}, y_{1}\right),\left(x_{2}, y_{2}\right) \in \mathbb{R}^{2} .
$$

For $\alpha \neq 0$, let us define the transformation

$$
\left\{\begin{array}{l}
X=x \\
Y=\sqrt{|\alpha|} y
\end{array}\right.
$$

then, equation (8) expressed in terms of $g(X, Y)=f(x, y)$ becomes

$$
\begin{aligned}
g\left(X_{1}, Y_{1}\right) g\left(X_{2}, Y_{2}\right) & =f\left(x_{1}, y_{1}\right) f\left(x_{2}, y_{2}\right) \\
& =f\left(x_{1} x_{2}+\alpha y_{1} y_{2}, x_{1} y_{2}+x_{2} y_{1}\right) \\
& =g\left(X_{1} X_{2}+\alpha \frac{Y_{1}}{\sqrt{|\alpha|}} \frac{Y_{2}}{\sqrt{|\alpha|}}, \sqrt{|\alpha|}\left(X_{1} \frac{Y_{2}}{\sqrt{|\alpha|}}+X_{2} \frac{Y_{1}}{\sqrt{|\alpha|}}\right)\right) \\
& =g\left(X_{1} X_{2}+\operatorname{sgn} \alpha Y_{1} Y_{2}, X_{1} Y_{2}+X_{2} Y_{1}\right) .
\end{aligned}
$$

Hence, in order to solve equation (8) it suffices to solve (10) and the following two equations

$$
\begin{aligned}
& f\left(x_{1}, y_{1}\right) f\left(x_{2}, y_{2}\right)=f\left(x_{1} x_{2}+y_{1} y_{2}, x_{1} y_{2}+x_{2} y_{1}\right), \\
& f\left(x_{1}, y_{1}\right) f\left(x_{2}, y_{2}\right)=f\left(x_{1} x_{2}-y_{1} y_{2}, x_{1} y_{2}+x_{2} y_{1}\right),
\end{aligned}
$$

where the domain of the variables is $\left(x_{1}, y_{1}\right),\left(x_{2}, y_{2}\right) \in \mathbb{R}^{2}$.

Every one of these cases is separately considered in the following three subsections.

2.1. The case $\alpha=1$. The transformation

$$
\left\{\begin{array}{l}
X=\frac{\sqrt{2}}{2}(x+y) \\
Y=\frac{\sqrt{2}}{2}(x-y)
\end{array}\right.
$$

reduces equation (12) to the following one:

$$
g\left(x_{1}, y_{1}\right) g\left(x_{2}, y_{2}\right)=g\left(x_{1} x_{2}, y_{1} y_{2}\right), \quad\left(x_{1}, y_{1}\right),\left(x_{2}, y_{2}\right) \in \mathbb{R}^{2} .
$$


In fact, setting $X_{k}=x_{k}+y_{k}, Y_{k}=x_{k}-y_{k}, k=1,2$, we have

$$
\begin{aligned}
X_{1} X_{2}+Y_{1} Y_{2} & =\frac{1}{2}\left(\left(x_{1}+y_{1}\right)\left(x_{2}+y_{2}\right)+\left(x_{1}-y_{1}\right)\left(x_{2}-y_{2}\right)\right) \\
& =x_{1} x_{2}+y_{1} y_{2}
\end{aligned}
$$

and, similarly,

$$
X_{1} Y_{2}+X_{2} Y_{1}=x_{1} x_{2}-y_{1} y_{2}
$$

hence, defining $f(X, Y)=g(x, y)$ we have

$$
\begin{aligned}
g\left(x_{1}, y_{1}\right) g\left(x_{2}, y_{2}\right) & =f\left(X_{1}, Y_{1}\right) f\left(X_{2}, Y_{2}\right)= \\
& =f\left(X_{1} X_{2}+Y_{1} Y_{2}, X_{1} Y_{2}+X_{2} Y_{1}\right) \\
& =f\left(x_{1} x_{2}+y_{1} y_{2}, x_{1} x_{2}-y_{1} y_{2}\right) \\
& =g\left(x_{1} x_{2}, y_{1} y_{2}\right) .
\end{aligned}
$$

In these last equalities, the bijectivity of the transformation (14) ensures that $\mathbb{R}^{2}$ is the domain of the variables $\left(x_{1}, y_{1}\right),\left(x_{2}, y_{2}\right)$.

Proposition 2. The general solution to equation (15) is given by

$$
g(x, y)=M_{1}(x) M_{2}(y), \quad x, y \in \mathbb{R},
$$

where $M_{1}, M_{2}: \mathbb{R} \rightarrow \mathbb{R}$ are two arbitrary multiplicative functions from $\mathbb{R}$ to $\mathbb{R}$.

Proof. Let $g$ be a solution to equation (15). Then, it is easily deduced that the functions $M_{1}$ and $M_{2}$ respectively defined by $M_{1}(x)=g(x, 1)$ and $M_{2}(x)=$ $g(1, x)$ are both multiplicative. For example, by setting $y_{1}=1=y_{2}$ in $(15)$, for $x_{1}, x_{2} \in \mathbb{R}$ we obtain

$$
M_{1}\left(x_{1}\right) M_{1}\left(x_{2}\right)=g\left(x_{1}, 1\right) g\left(x_{2}, 1\right)=g\left(x_{1} x_{2}, 1\right)=M_{1}\left(x_{1} x_{2}\right) ;
$$

i.e., $M_{1}$ is a multiplicative function.

Using again equation (15), we see that

$$
g(x, y)=g(x, 1) g(1, y)=M_{1}(x) M_{2}(y), \quad x, y \in \mathbb{R} .
$$

The argument is completed by observing that equation (15) is solved by every function of the form (16) with $M_{1}$ and $M_{2}$ two given multiplicative functions.

Proposition 3. The general solution to equation (12) is given by

$$
f(x, y)=M_{1}(x+y) M_{2}(x-y), \quad x, y \in \mathbb{R},
$$

where $M_{1}, M_{2}: \mathbb{R} \rightarrow \mathbb{R}$ are two arbitrary multiplicative functions from $\mathbb{R}$ to $\mathbb{R}$.

Proof. From the previous discussion we see that the general solution to equation (12) is given by

$$
\begin{aligned}
f(x, y) & =M_{1}\left(\frac{\sqrt{2}}{2}(x+y)\right) M_{2}\left(\frac{\sqrt{2}}{2}(x-y)\right) \\
& =c M_{1}(x+y) M_{2}(x-y),
\end{aligned}
$$


for a certain real constant $c$. Then, by inserting the last member of these equalities in equation (12) we obtain

$$
c^{2}=c
$$

i.e., $c=0$ or $c=1$. The first value gives the trivial solution (also obtained by setting $M_{1}=0$ in (17)), meanwhile the second one gives (17).

2.2. The case $\alpha=0$. Setting $x_{1}=0=x_{2}$ in (10) yields

$$
f\left(0, y_{1}\right) f\left(0, y_{2}\right)=f(0,0), \quad y \in \mathbb{R}
$$

in particular, for $y_{1}=y=y_{2}$ :

$$
f^{2}(0, y)=f(0,0), \quad y \in \mathbb{R} .
$$

Then

$$
f^{2}(0,0)=f(0,0)
$$

so that $f(0,0)=0$ or $f(0,0)=1$.

Since

$$
f(0,0) f(x, y)=f(0,0), \quad(x, y) \in \mathbb{R}^{2},
$$

we see that $f(x, y) \equiv 1$ when $f(0,0)=1$.

Now, putting aside the case in which $f(0,0)=1$, the equality $f(0,0)=0$ holds; then $f(0, y)=0$ by (19). In this case we have

$$
f(x, y)=f\left(0, y_{0}\right) f(x, y)=f\left(0, y_{0} x\right)=0, \quad(x, y) \in \mathbb{R}^{2} .
$$

Thus, we have proved the following result.

Proposition 4. The general solution to the equation (10) is composed by the trivial solutions $f \equiv 0$ or $f \equiv 1$.

Proof. See the previous discussion.

It should be noted that the general solution to equation (10) may be a richer set if solved on different domains for the variables. For example, we will solve it for the domain $(x, y) \in \mathbb{R}^{2}$ with $x \neq 0$.

Setting $y_{1}=0=y_{2}$ in equation (10) yields

$$
f\left(x_{1}, 0\right) f\left(x_{2}, 0\right)=f\left(x_{1} x_{2}, 0\right), \quad x_{1}, x_{2} \in \mathbb{R}^{2} .
$$

Furthermore, the equation

$$
f\left(1, y_{1}\right) f\left(1, y_{2}\right)=f\left(1, y_{1}+y_{2}\right), \quad y_{1}, y_{2} \in \mathbb{R}^{2}
$$

is obtained by setting $x_{1}=1=x_{2}$ in (10). (20) and (21) are Cauchy's equations whose general solutions are given respectively by

$$
f(x, 0)=M(x), \quad x \in \mathbb{R}
$$

and

$$
f(1, y)=\exp (A(y)), \quad y \in \mathbb{R},
$$

with $M$ a multiplicative function and $A$ an additive one. 
Now observe that, for every pair $(x, y) \in \mathbb{R}^{2}$ with $x \neq 0$ is

$$
f(x, y)=f(x, 0) f(1, y / x) .
$$

Thus, from (22), (23) and (24) we obtain

$$
f(x, y)=M(x) \exp \left(A\left(\frac{y}{x}\right)\right), \quad(x, y) \in \mathbb{R}^{2}, \quad x \neq 0 .
$$

That (25) is the general solution to equation (10) for $(x, y) \in \mathbb{R}^{2}, x \neq 0$, is seen by replacing it in the equation.

2.3. The case $\alpha=-1$. When $\alpha=-1$, for $\zeta \in \mathbb{C}$ of the form $\zeta=x+i y$ let us define

$$
\phi(\zeta)=f(x, y), \quad(x, y) \in \mathbb{R}^{2} .
$$

In terms of $\phi$, equation (8) then becomes

$$
\phi\left(\zeta_{1}\right) \phi\left(\zeta_{2}\right)=\phi\left(\zeta_{1} \zeta_{2}\right)
$$

where $\zeta_{1}, \zeta_{2} \in \mathbb{C}=\mathbb{R}+i \mathbb{R}$.

Two simple observations are in order in connection with a solution $\phi$ to equation (26). First, by setting $\zeta_{1}=\zeta=\zeta_{2}$, we obtain $\phi\left(\zeta^{2}\right)=\phi^{2}(\zeta) \geq 0$ for every $\zeta \in \mathbb{C}$; and hence $\phi \geq 0$. Moreover, if $\phi\left(\zeta_{0}\right)=0$ for a given $\zeta_{0} \in \mathbb{C}$; then $\phi\left(\zeta_{0} \zeta\right)=\phi\left(\zeta_{0}\right) \phi(\zeta)=0$ for every $\zeta \in \mathbb{C}$. Therefore, if a solution $\phi$ of equation (26) vanishes in a point; then it vanishes in every point and therefore it is the trivial zero solution $\phi(\zeta) \equiv 0$.

Now, since $\zeta_{1} \zeta_{2} \in \mathbb{R}$ when $\zeta_{1}, \zeta_{2} \in \mathbb{R}$, it is seen that

$$
\phi\left(r_{1}\right) \phi\left(r_{2}\right)=\phi\left(r_{1} r_{2}\right), \quad r_{1}, r_{2} \in \mathbb{R} .
$$

The solution to this (multiplicative Cauchy's) equation is given (cf. for instance page 31 of [1]) by a multiplicative function

$$
\phi(r)=M(r), \quad r \in \mathbb{R} .
$$

Now, replacing in (26) the polar decomposition $\zeta=\sqrt{x^{2}+y^{2}} \exp (i \theta)$ with $\theta=\arg \zeta$, and taking into account $(27)$, we obtain

$$
\begin{aligned}
\phi(\zeta) & =\phi\left(\sqrt{x^{2}+y^{2}} \exp (i \arg \zeta)\right) \\
& =\phi\left(\sqrt{x^{2}+y^{2}}\right) \phi(\exp (i \arg \zeta))=M\left(\sqrt{x^{2}+y^{2}}\right) \phi(\exp (i \arg \zeta)) .
\end{aligned}
$$

Now, for $\theta_{1}, \theta_{2} \in \mathbb{R}$ we have

$$
\phi\left(\exp \left(i \theta_{1}\right)\right) \phi\left(\exp \left(i \theta_{2}\right)\right)=\phi\left(\exp \left(i \theta_{1}\right) \exp \left(i \theta_{2}\right)\right)=\phi\left(\exp \left(i\left(\theta_{1}+\theta_{2}\right)\right)\right) ;
$$

thus, in terms of

$$
\psi(\theta)=\phi(\exp (i \theta)), \quad \theta \in \mathbb{R},
$$

the equality between the first and third members of (28) becomes

$$
\psi\left(\theta_{1}+\theta_{2}\right)=\psi\left(\theta_{1}\right) \psi\left(\theta_{2}\right), \theta_{1}, \theta_{2} \in \mathbb{R} .
$$


The general solution to (exponential Cauchy's) equation (29) is given (cf. for instance page 29 of [1]) by an exponential function $\psi(\theta)=\exp A(\theta), \theta \in \mathbb{R}$, with $A: \mathbb{R} \rightarrow \mathbb{R}$ an additive function.

In conclusion, $\phi \equiv 0$ or

$$
\phi(\zeta)=M\left(\sqrt{x^{2}+y^{2}}\right) \exp (A(\arg (\zeta)))
$$

where $M$ and $A$ are, respectively, a multiplicative and an additive functions.

Proposition 5. The general solution $f: \mathbb{R}^{2} \rightarrow \mathbb{R}$ to equation (13) are the trivial one $f \equiv 0$ and those of the form

$$
f(x, y)=M\left(\sqrt{x^{2}+y^{2}}\right) \exp (A(\operatorname{atan}(y / x))
$$

for a pair of arbitrary (non vanishing) multiplicative $M$ and additive $A$ functions from $\mathbb{R}$ to $\mathbb{R}$.

Proof. A substitution of (30) in (13) shows that (30) actually solves equation (13). After the previous discussion, it is concluded that $f$ given by (30) is the general solution to (13).

2.4. Proof of Theorem 1. The proof of Theorem 1 is easily derived from Propositions (3), (4) and (5) after applying transformation (11) as described at the beginning of the section.

\section{Final REMARKS: INTEgRAL BASIS OF QUADRATIC NUMBER FIELDS AND A SEEMINGLY MORE GENERAL FUNCTIONAL EQUATION}

It might be thought that a functional equation more general than (8) can be suggested by the selection of a different basis for the field $\mathbb{Q}(\sqrt{\alpha})$. As shown in the lines that follows, this is not the case.

In fact, take for simplicity the case of a square-free integer $\alpha>0$. As it is wellknown, for some values of $\alpha$ the ring of algebraic integers of this number field is larger than $\mathbb{Z}[\alpha]$, in fact (for some $\alpha$ ) it is a free $\mathbb{Z}$-module of rank 2 generated by 1 and $\frac{1+\sqrt{\alpha}}{2}$. If we consider algebraic integers in this field it is thus natural to take the basis $\left(1, \frac{1+\sqrt{\alpha}}{2}\right)$ and when multiplying two elements we want to consider the functional equation describing how the coordinates (with respect to this basis) behave under this product. Explicitly, the product formula is given by

$$
\begin{aligned}
\left(x_{1}+\frac{1+\sqrt{\alpha}}{2} y_{1}\right)\left(x_{2}+\frac{1+\sqrt{\alpha}}{2} y_{2}\right)= & \left(x_{1} x_{2}+\frac{\alpha-1}{4} y_{1} y_{2}\right) \\
& +\frac{1+\sqrt{\alpha}}{2}\left(x_{1} y_{2}+x_{2} y_{1}+y_{1} y_{2}\right) .
\end{aligned}
$$


Then, the functional equation associated to this formula is

$$
f\left(x_{1}, y_{1}\right) f\left(x_{2}, y_{2}\right)=f\left(x_{1} x_{2}+\frac{\alpha-1}{4} y_{1} y_{2}, x_{1} y_{2}+x_{2} y_{1}+y_{1} y_{2}\right)
$$

where $\left(x_{1}, y_{1}\right),\left(x_{2}, y_{2}\right) \in \mathbb{R}^{2}$. The appearance of this equation differs from that one of (8) but it will be seen that a simple change of variables reduces one to the other. In fact, just taking $x_{i}^{\prime}=x_{i}+\frac{y_{i}}{2}, y_{i}^{\prime}=y_{i}$, setting $f(x, y)=f\left(x^{\prime}-\frac{y^{\prime}}{2}, y^{\prime}\right)=$ $g\left(x^{\prime}, y^{\prime}\right)$ and writing the functional equation for $g$ that follows from the one for $f$, we obtain

$$
g\left(x_{1}^{\prime}, y_{1}^{\prime}\right) g\left(x_{2}^{\prime}, y_{2}^{\prime}\right)=g\left(x_{1}^{\prime} x_{2}^{\prime}+\frac{\alpha}{4} y_{1}^{\prime} y_{2}^{\prime}, x_{1}^{\prime} y_{2}^{\prime}+x_{2}^{\prime} y_{1}^{\prime}\right)
$$

which coincides with equation (8) with a parameter $\alpha / 4$ instead of $\alpha$. Of course, the general solution to equation (31) is deduced from the general solution to (8) by reversing the change of coordinates above. In this way, we obtain the general solution of the functional equation for $f$, which, as expected, is the following:

$$
f(x, y)=M_{1}\left(x+\frac{1+\sqrt{\alpha}}{2} y\right) M_{2}\left(x+\frac{1-\sqrt{\alpha}}{2} y\right)
$$

where $M_{1}$ and $M_{2}$ are two arbitrary multiplicative functions from $\mathbb{R}$ to $\mathbb{R}$.

\section{ACKNowledgements}

The bulk of this paper was completed during a sojourn of the first author at the Centre de Recerca Matematica, Universitat Autònomoma de Barcelona.

\section{REFERENCES}

[1] J. Aczél, J. Dhombres, Functional Equations in Several Variables (with applications to mathematics, information theory an to the natural and social sciences), Cambridge Univ. Press, Cambridge, 1989.

[2] Leonardo of Pisa, The Book of Squares. An annotated translation of the Liber Quadratorum into modern English by L. E. Siegler, Academic Press, 1987.

[3] Soon-mo Jung, Jae-Hyeong Bae, Some functional equations originating from number theory, Proc. Indian Acad. Sci. (Math. Sci.), Vol. 113, No. 2., May 2003, 91-98. 
LuCio R. Berrone

Consejo Nacional de Investigaciones Científicas y Técnicas (CONICET)

Laboratorio DE Acústica y Electroacústica

Facultad de Ciencias Exactas, Ing. y Agrim.

Universidad Nacional DE Rosario

RIOBAMBA 245 BIS

(2000) Rosario, Argentina

E-mail address: berrone@fceia.unr.edu.ar

Luis Dieulefait

Departament D'Algebra i Geometria

UNIVERSITAT DE BARCELONA

Gran Via de les Corts Catalanes 585

(08007) Barcelona, Spain

E-mail address: ldieulefait@ub.edu 